\title{
Non-linearity of LEDs for VLC IoT applications
}

\author{
Muhammad Sarmad Mir \\ IMDEA Networks Institute \\ Madrid, Spain \\ Ander Galisteo \\ IMDEA Networks Institute \\ Madrid, Spain
}

\author{
Borja Genoves Guzman \\ IMDEA Networks Institute \\ Madrid, Spain \\ Domenico Giustiniano \\ IMDEA Networks Institute \\ Madrid, Spain
}

\begin{abstract}
Visible light communication (VLC) is a promising technology to satisfy the increasing demand of wireless communication services. The generalized idea of modulating the signal within the light-emitting diode (LED) linear region is considered the de-facto approach for high-speed communication. This paper shows that this is not the most appropriate choice for the Internet of Things (IoT) applications, where the communication range is of greater importance once a sufficient communication rate is reached. In order to improve the communication range, we propose to exploit the full non-linear region of an LED transceiver while maintaining constant illumination. We show that working in the full LED region can provide received electrical power gains in the mobile IoT tag of around $15 \mathrm{~dB}$. We achieve this without affecting the communication rate for IoT applications, and at the penalty of about $10 \%$ increase in the total energy consumption of LEDs. We further demonstrate that exploiting the non-linear region of LEDs is beneficial for mobile tags that employ a solar cell for receiving data at high data rates.
\end{abstract}

\section{CCS CONCEPTS}

- Computer systems organization $\rightarrow$ Embedded hardware; • Hardware $\rightarrow$ Wireless devices.

\section{KEYWORDS}

LED non-linearity, visible light communication, internet-of-things, OpenVLC

\section{ACM Reference Format:}

Muhammad Sarmad Mir, Borja Genoves Guzman, Ander Galisteo, and Domenico Giustiniano. 2020. Non-linearity of LEDs for VLC IoT applications. In Light Up the IoT (LIOT '20), September 21, 2020, London, United Kingdom. ACM, New York, NY, USA, 6 pages. https://doi.org/10.1145/3412449.3412552

\section{INTRODUCTION}

Nowadays, light-emitting diodes (LEDs) are commonly used for generalized lighting applications due to many advantages over the traditional incandescent and fluorescent bulbs, such as its higher energy efficiency, longer lifespan, smaller size and better safety features [16]. An LED can also be modulated at high speed, which makes it suitable for visible light communication (VLC) [10]. VLC

LIOT '20, September 21, 2020, London, United Kingdom

(C) 2020 Association for Computing Machinery.

This is the author's version of the work. It is posted here for your personal use. Not for redistribution. The definitive Version of Record was published in Light Up the IoT (LIOT '20), September 21, 2020, London, United Kingdom, https://doi.org/10.1145/ 3412449.3412552. combines illumination with data transfer and aims to retrofit the same lighting infrastructure for high-speed and power-efficient data communication. In the last few years, interest has sparked in the research community to investigate VLC for the Internet of Things (IoT) $[11,13-15,17]$. The aim of this paper is to demystify the use of only the linear LED region. This choice is inherited from high-speed VLC and is motivated mainly by the fact that it minimizes the time response of LEDs [9]. However, we demonstrate in this work that it is not the most appropriate approach for IoT applications, as the optimal operational point in the trade-off between communication rate and range is different with respect to high-speed communication. For instance, better communication range is desired for the application of VLC in industrial IoT, whose height in production floors is typically higher than in-home scenarios [12]. It is also required for outdoor applications, for instance for VLC IoT in light posts.

An LED is a unidirectional current-driven conductive device whose brightness is dependent on the forward current and it is controlled by the forward voltage. Thus, an LED as a VLC transmitter follows an intensity modulation. The LED presents a non-linear transfer function in the relation between the forward voltage and the output luminous flux. To avoid clipping issues, distortions and to increase the response speed in the transmitted signal, the forward current must be DC-biased and scaled accordingly in order to accommodate the signal within the linear region of the LED [6]. However, the limited linear region corresponds to smaller peakto-peak transmit signal swing that reduces the received electrical power at the VLC receiver, causes reduction in the communication range and increases the vulnerability to noise and interference. Despite this, VLC systems for IoT presented in the literature work in the LED linear region [2,3]. The LED light intensity is modulated using on-off keying (OOK) and the LED is operated with a bias current to achieve the maximum swing in the linear region for high and low signals.

In this work, we design and demonstrate a low-cost scheme to communicate and exploit the full non-linear region of LEDs with OOK modulation. Our experimental study shows that working in the full LED region can provide received electrical power gains in the IoT tag of around $15 \mathrm{~dB}$, without affecting the communication rate for IoT applications. This is at the expense of increasing the total transmitter power consumption in the LED deployed of about $10 \%$. This cost is acceptable: we show analytically that as we move from the linear region to the non-linear region, the SNR at the receiver increases faster than the power consumption at the LED transmitter. We further demonstrate that exploiting the full region of LEDs is beneficial also for receivers that use a solar cell for 


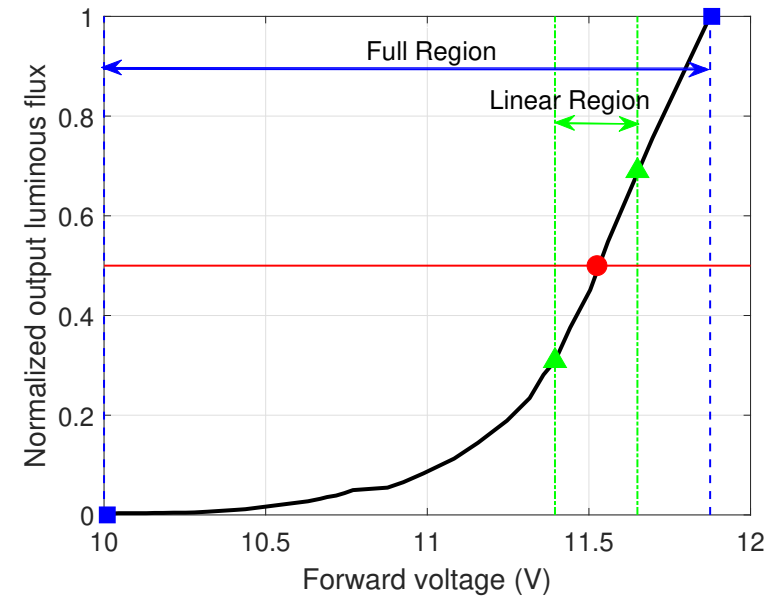

Figure 1: Transfer function of the LED model Cree XLamp XHP35A-00-0000-0D0HC40E7 [1]. Our full-region operation design and the typical linear-region operation design of LEDs are also represented.

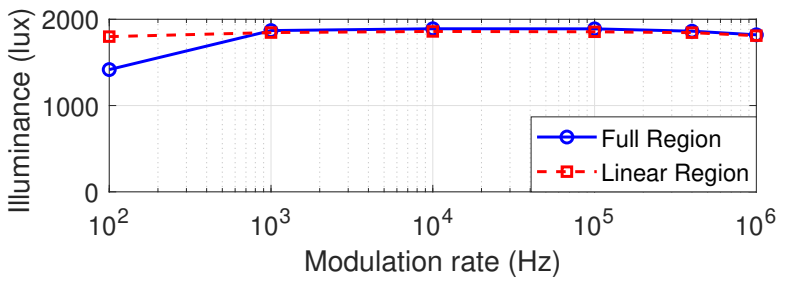

Figure 2: Illuminance provided at a distance of $60 \mathrm{~cm}$.

receiving data, as working in the non-linear region allows solar cells to receive data at higher data rates.

\section{DESIGN}

Typically, LEDs are modulated within its linear region around the bias working point. An illustration is represented in Fig. 1, where we show the transfer function of one LED model and indicate the bias point with a red circle. The bias point is experimentally obtained as the midpoint of the possible output luminous flux values. We derive the transfer function using a modified OpenVLC board where we change the series resistance to the LED using a potentiometer [7].

Fig. 1 illustrates the two approaches we compare, 'Full Region' (delimited by square markers) and 'Linear Region' (delimited by triangle markers). Operating in the linear region allows for fast LED responsiveness, which is ideal for high-data rate communications [9]. Yet, this approach results in small peak-to-peak current swing $\left(I_{\mathrm{sw}}\right)$. As the current is approximately proportional to the light received at the destination, small swings negatively affect the communication range. As IoT communication has lower demands in terms of data rate, we work with two levels of light intensity to guarantee low-power processing on the receiver side. As in traditional OOK, we associate bit 1 and 0 to high and low intensity, respectively, and we operate with the same bias current in order

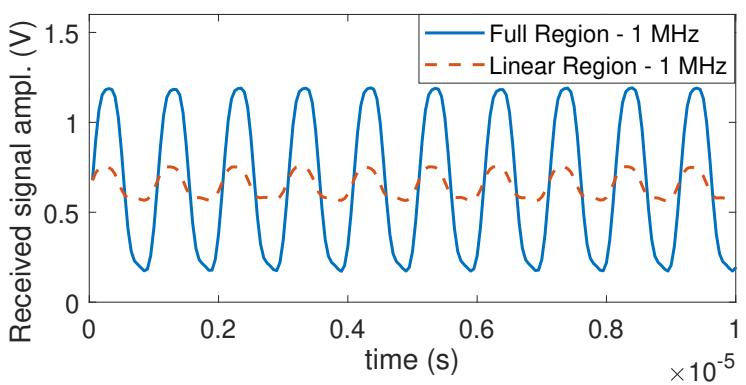

Figure 3: Capacitance effect at high modulation rate.

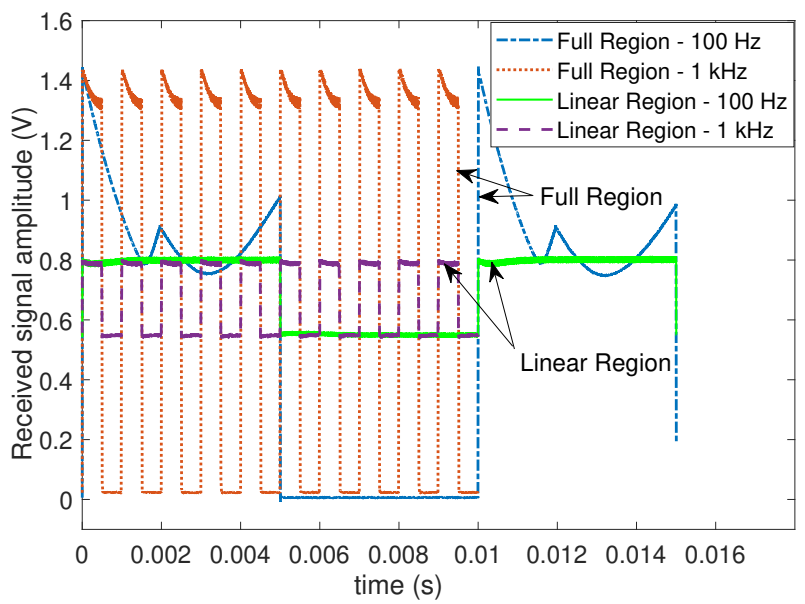

Figure 4: Demonstration of temperature issue by plotting time-domain received signals at a distance of $1 \mathrm{~m}$ when using a QSD2030 plastic silicon photodiode and a typical transimpedance amplifier. Captures when transmission is done with the two approaches and at different modulation rates are represented.

to guarantee the same illumination level. This is shown in the red solid line in Fig. 1 at a normalized output luminous flux of 0.5. In fact, the design of a LiFi front-end in the bulb must provide a constant average illumination and avoid flickering, complying with the illumination regulation. However, in contrast to past designs, we select the driving voltage to maximize the communication range.

In Fig. 2 we experimentally demonstrate that the two approaches have the same output luminous flux. The study is conducted measuring the illuminance at a distance of $60 \mathrm{~cm}$. We note that both approaches provide the same illuminance regardless of the OOK modulation rate. There are two exceptions: i) the highest modulation rate $(1 \mathrm{MHz})$, where the LED capacitance effect makes illumination slightly decrease, as demonstrated in Fig. 3, where the transmit signal is not square (low and high levels are not achieved) because the response speed of LED is slower than modulation rate, ii) the lowest modulation rate $(100 \mathrm{~Hz})$ where the illuminance provided by full region approach decreases likely due to the thermal issue that arises for keeping the LED on at higher power. In fact, when 


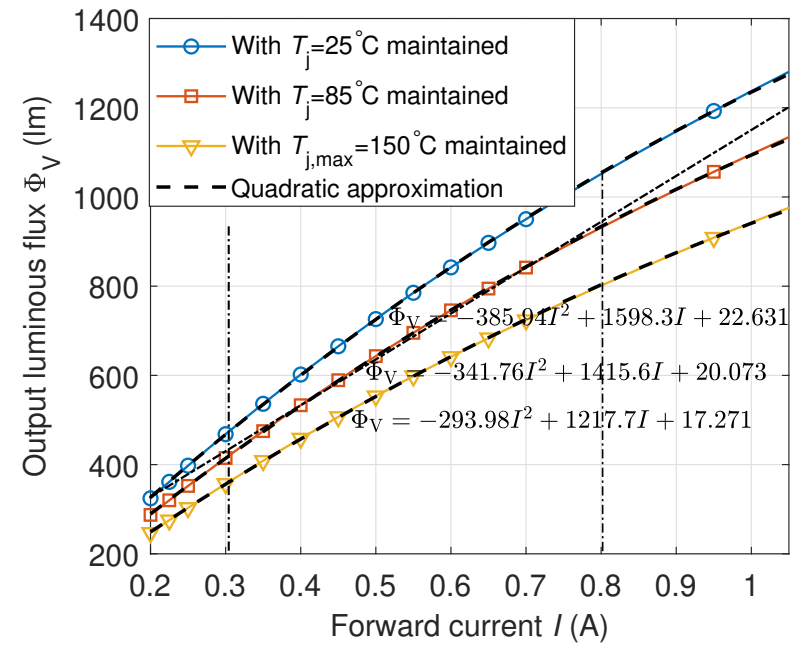

Figure 5: Non-linear (concave) function of the output luminous flux against the forward current for different junction temperatures of LED model Cree XLamp XHP35A-00-00000D0HC40E7.

working at the maximum forward voltage, the LED may suffer from a junction temperature increase that, if it is maintained over the time, could damage the LED and malfunction.

This effect is represented in Fig. 4. The figure shows the timedomain received signal after a trans-impedance amplifier (TIA) and a photodiode (PD) QSD2030 (first stage of the receiver in an OpenVLC board). The figure shows that the received signal amplitude becomes unstable at very low modulation rate $(100 \mathrm{~Hz})$. Thus, as a precaution for new designs, thermal management is an issue to consider especially when working with high brightness LEDs. Fortunately, this only happens at very low modulation rates $(100 \mathrm{~Hz})$, that is below the typical desired transmission rates. To avoid this issue, the transmission rate in our experiments are taken from a modulation rate of $1 \mathrm{kHz}$ onwards.

\section{ANALYSIS}

In joint illumination and communication systems (JIC), as in VLC systems, the power consumption is higher in comparison with only illumination systems. In other terms, modulating the input signal of an LED increases the energy consumption. This consumption for communication is mainly due to two reasons, the concavity of the forward current and output luminous flux function and the convexity of the forward current and the electrical power of LED function.

1. Concavity of the forward current and output luminous flux function. The $I-\Phi_{\mathrm{V}}$ curve can be approximated by a concave quadratic function as:

$$
\Phi_{\mathrm{V}} \approx a I^{2}+b I+c,
$$

where the parameters $a, b$ and $c$ are the polynomial coefficients. Note that $a<0$, which demonstrates that the curve is concave. We illustrate this concavity in the experimental tests in Fig. 5. When a modulation signal $s(t)$ is superimposed to the current $I$, that is
$I(t)=[1+s(t)] \cdot I$, the averaged output luminous flux is given by [5]:

$$
\begin{aligned}
E\left[\Phi_{\mathrm{V}}\right] & =\Phi_{\mathrm{V}}(I)+\left(2 a I^{2}+b I\right) E[s(t)]+a I^{2} E\left[s^{2}(t)\right] \\
& \approx \Phi_{\mathrm{V}}(I)+a I^{2} E\left[s^{2}(t)\right],
\end{aligned}
$$

where $E\left[s^{2}(t)\right]=\left(I_{\mathrm{sw}} / 2\right)^{2}$ if an OOK modulation scheme is employed, and $I_{\mathrm{sw}}$ is the peak-to-peak current swing of the forward current. Note that the larger the $I_{\mathrm{sw}}$ value, the lower the average output luminous flux is. Thus, when a JIC system is employed, the average output luminous flux $E\left[\Phi_{\mathrm{V}}\right]$ decreases. If a larger $E\left[\Phi_{\mathrm{V}}\right]$ value is desired by using OOK, we must increase the DC-bias value by accommodating low and high levels, which means an increased electrical power consumption.

2. Convexity of the forward current and electrical power of LED function. The $I-P_{\text {LED }}$ curve is typically approximated as [5]:

$$
P_{\mathrm{LED}}=\frac{n k T}{q} \cdot I \cdot \ln \left(\frac{I}{I_{\mathrm{S}}}+1\right)+R_{\mathrm{L}} I^{2}
$$

where $n$ is the ideality factor, $k$ is Boltzmann constant, $T$ denotes the temperature, $q$ is the electron charge, $I_{\mathrm{S}}$ is the saturation current of the LED and $R_{\mathrm{L}}$ is the LED DC resistance. By approximating (3) with Taylor series [3], the average power consumed on communication can be written as:

$$
P_{\mathrm{LED}, \mathrm{c}}=r \cdot\left(\frac{I_{\mathrm{SW}}}{2}\right)^{2},
$$

where $r=\frac{n k T}{2 q I_{\mathrm{DC}}}+R_{\mathrm{L}}$. Note that, again here, a larger $I_{\mathrm{Sw}}$ value means an increase electrical power consumption.

When comparing two OOK approaches (non-linear and linear) that provide the same $E\left[\Phi_{\mathrm{V}}\right]$ as those under study in this paper, the concavity of the $I-\Phi_{\mathrm{V}}$ function will not affect the energy consumption. However, as shown in (4), the peak-to-peak current swing influences the energy consumption as well as the communication performance given by the signal-to-noise ratio (SNR) as:

$$
\gamma=\frac{P_{\mathrm{r}, \mathrm{elec}}}{\sigma_{\mathrm{n}}^{2}}=\frac{\left(R P_{\mathrm{r}, \mathrm{opt}}\right)^{2}}{\sigma_{\mathrm{n}}^{2}}=\frac{\left(R \cdot \eta_{\mathrm{LED}} \cdot h \cdot r \cdot\left(\frac{I_{\mathrm{sw}}}{2}\right)^{2}\right)^{2}}{B \cdot N_{0}},
$$

where $R$ is the PD's responsivity, $P_{\mathrm{r}, \text { elec }}$ and $P_{\mathrm{r}, \mathrm{opt}}$ denote the electrical and optical received power, respectively, the noise power is represented by $\sigma_{\mathrm{n}}^{2}=B \cdot N_{0}$, where $B$ is the channel bandwidth and $N_{0}=N_{0, \text { th }}+N_{0, \mathrm{~s}}$ is the power spectral density of noise, composed by the power spectral densities of thermal noise $N_{0 \text {,th }}$ and shot noise $N_{0, \mathrm{~s}}$. Note that thermal noise does not depend on the received signal, but shot noise is proportional to optical received power [4] according to $N_{0, \mathrm{~s}}=2 q P_{\mathrm{r}, \mathrm{opt}} R$, where $P_{\mathrm{r} \text {,opt }}$ is the optical received power. Finally, $\eta_{\mathrm{LED}}$ is the electrical to optical conversion coefficient and $h$ is the free-space channel path loss. From (4) and (5), $P_{\mathrm{LED}, \mathrm{c}} \propto\left(I_{\mathrm{sw}}\right)^{2}$ whereas $\gamma \propto\left(I_{\mathrm{SW}}\right)^{4}$ as long as the system is limited by thermal noise rather than shot noise, which is typical in indoor scenarios. The consequence is that, as we move from the linear region to the non-linear region, the SNR at the receiver increases faster than the power consumption at the LED transmitter.

Example. When working with $\mathrm{OOK}$ and following the $I-\Phi_{\mathrm{V}}$ curve for $T_{\mathrm{j}}=85^{\circ} \mathrm{C}$ of Fig. 5 , the maximum peak-to-peak current 


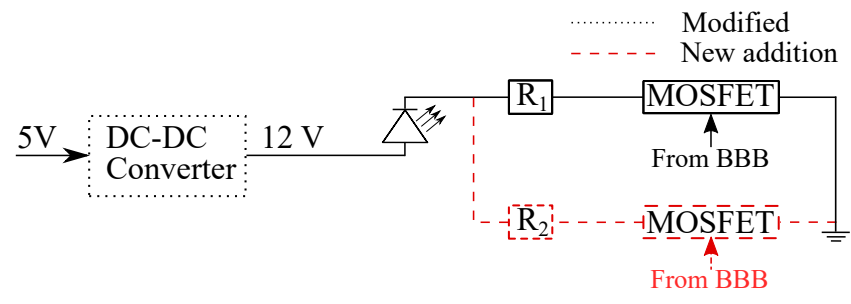

Figure 6: Block diagram of modified OpenVLC

swing is $I_{\mathrm{sw}, \max }=0.85 \mathrm{~A}$, providing $E\left[\Phi_{\mathrm{V}}\right]=709.59 \mathrm{~lm}$. The analogous OOK system working within the linear region providing the same $E\left[\Phi_{\mathrm{V}}\right]$ is the one represented by dash-dotted lines in Fig. 5, with a current swing of $I_{\mathrm{sw}, \text { linear }}=0.5 \mathrm{~A}$. Thus, modulating the OOK signal within the linear region means decreasing the electrical power allocated for communication purposes to $34 \%$. However, the SNR is reduced to $12 \%$. Considering that the electrical power consumption for communication is a low portion of the total electrical power (as most of it comes from the illumination purpose), the total electrical power when working within the linear region is not decreased as much as up to $34 \%$. Furthermore, the SNR reduction up to a $12 \%$ may be critical for communication. In conclusion, due to these theoretical reasons, modulating the OOK signal in the whole allowable region is preferable rather than only in the linear range. In the next section, we verify experimentally this analysis.

\section{EVALUATION}

In this section, we perform a set of experiments to compare the performance obtained when a light signal is modulated with an OOK scheme only using the linear region of an LED, versus the performance when the full range is used.

\subsection{Experimental setup}

The platform used for the experiments is based on OpenVLC 1.3 [7], which is a low-cost open-source research-oriented platform. In both approaches (full region and linear region) the cape of OpenVLC1.3 is modified to change the high and low levels of the OOK modulated signal. In OpenVLC1.3 the DC-DC converter is used to step up $5 \mathrm{~V}$ input to $11.2 \mathrm{~V}$ to operate the LED. Here, the output of the DC-DC converter is modified to give $12 \mathrm{~V}$ to LED in order to explore its full range. In OpenVLC1.3 transmitter, one current path is used to transmit both high and low signal levels in OOK by toggling the I/O pin of BeagleboneBlack (BBB). In this modified version, a new current path is added to independently set the high level $\left(V_{\mathrm{H}}\right)$ and low level $\left(V_{\mathrm{L}}\right)$ using the series resistor $\left(R_{1}\right.$ and $\left.R_{2}\right)$ with LED in each path, as shown in Fig. 6. The MOSFET switch is used to enable one path at a time using BBB I/O pins. In the full region approach, a $0 \Omega$ resistor is used to set $V_{\mathrm{H}}$ at $11.87 \mathrm{~V}$ and a $10 \mathrm{k} \Omega$ resistor is used to set $V_{\mathrm{L}}$ below the turn-on voltage $(10 \mathrm{~V})$. In the linear region approach, $V_{\mathrm{H}}$ is set to $11.6 \mathrm{~V}$ using $1.2 \Omega$ and $V_{\mathrm{L}}$ is set to $11.4 \mathrm{~V}$ using $5 \Omega$ series resistor.

The experimental setup is shown in Fig. 7. We use Saleae logic 8 analyser to record data and Extech SDL400 lux meter to measure the illumination.

\subsection{Communication link performance}

The received electrical average power versus the distance between transmitter and receiver is depicted in Fig. 8a. The received electrical power is computed as the power of the received voltage signal without DC component measured just after the Trans-Impedance Amplifier (TIA), and then computed in $\mathrm{dBm}^{1}$. As expected, the larger the distance, the lower the received electrical average power is, which is proportional to $d^{-4}$ as shown in eq. (5) considering that the free-space channel gain $h$ is proportional to $d^{-2}$. A gain in the received electrical power of around $15 \mathrm{~dB}$ is obtained by using the full region of the LED in comparison to only using the linear region, which allows achieving higher modulation rates and distance. Results for every approach are similar except for at $1000 \mathrm{kHz}$, as illuminance slightly decreases due to the LED capacitance effect (cf. Fig. 2 and Fig. 3).

We then represent the SNR in Fig. $8 \mathrm{~b}$ as a function of the distance between the transmitter and the receiver, for different modulation rates and using the two approaches under study. We observe that the SNR decreases as the modulation rate increases due to the noise power increment, as indicated by eq. (5). If the rest of parameters in eq. (5) were independent of the modulation rate, we would observe a difference of $10 \mathrm{~dB}$ among curves with different rates, as we see when comparing curves at $10 \mathrm{kHz}$ and $100 \mathrm{kHz}$. However, a difference of $10 \mathrm{~dB}$ is not obtained when comparing curves at $1 \mathrm{kHz}$ and $10 \mathrm{kHz}$, or at $100 \mathrm{kHz}$ and $1000 \mathrm{kHz}$, due to temperature issues at the transmitter (see Fig. 4) and the capacitance effect of LED (see Fig. 3), respectively. Full region approach always provides a higher SNR than a linear region approach, except for when using $1 \mathrm{kHz}$ at a short distance, as LED could be malfunctioning due to the high temperature. Generally, the gain that the full region approach provides in comparison to the full region approach is at least $2 \mathrm{~dB}$, and higher at larger distances and low rates because the low peak-to-peak transmit signal swing of the linear region approach limits the achievable distance. The longer the distance, the lower the SNR is due to the electrical received power degradation. At high modulation rates, the SNR does not degrade with distance as much as at lower rates, because the system is limited by the effect that the bandwidth produces in the noise, rather than the degradation produced by the received optical power that only depends on the distance.

\subsection{Energy consumption}

The energy consumption is an important figure of metric in IoT systems, as the devices must consume as low as possible in order to boost its performance duration and minimize the carbon footprint. This section evaluates the energy consumption of both approaches when transmitting at different modulation rates, as represented in Fig. 9. The modulation rate does not affect the power consumption considerably. The full region approach consumes around $0.3 \mathrm{~W}$ larger than linear region approach, which is consistent with the analysis presented in Section 3, where (4) indicates that the power consumed by the communication is proportional to $I_{\mathrm{sw}}^{2}$, where $I_{\mathrm{sw}}$ is the peak-to-peak current swing of the electrical transmit signal. Assuming that the forward current is proportional to the forward

${ }^{1}$ It is computed as $\frac{1}{R T} \int_{0}^{T} v^{2}(t) \mathrm{d} t$ assuming unitary resistance $R$ and it is equivalent to the variance of the electrical signal. 

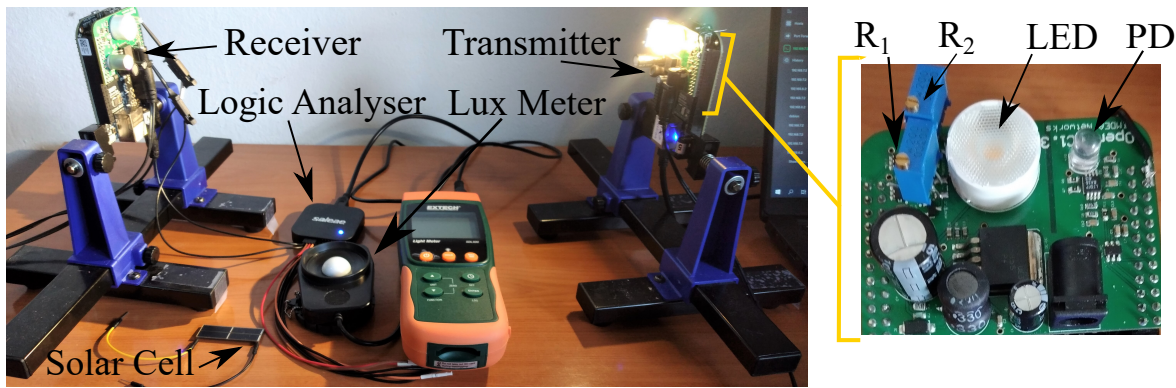

Figure 7: Experimental Setup

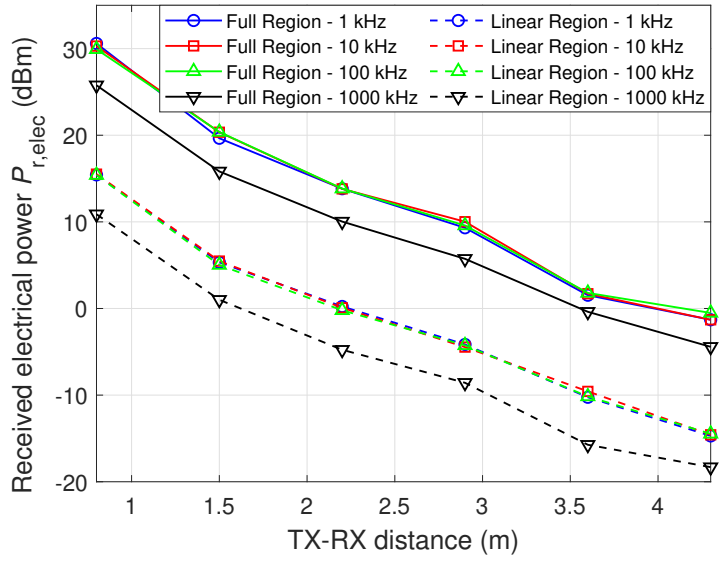

(a) Received electrical power versus distance.

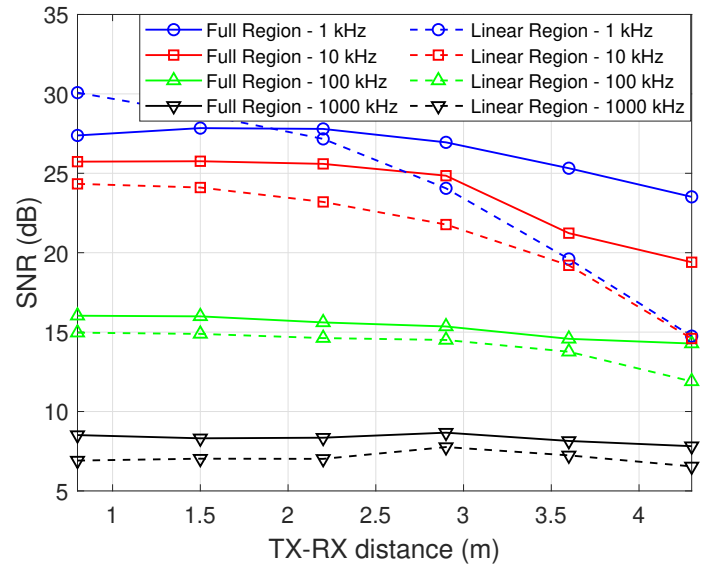

(b) SNR versus distance.

Figure 8: Metrics of the received signal measured after TIA.

voltage, the energy consumed by the communication action will be also proportional to $V_{\mathrm{sw}}^{2}$, where $V_{\mathrm{sw}}$ is peak-to-peak voltage swing across the LED. According to Fig. 1, we know that $V_{\mathrm{sw}}$ of full region approach is $V_{\mathrm{sw} \text {,full }}=11.87-10=1.87 \mathrm{~V}$, whereas the linear region approach modulates the transmit signal within $V_{\mathrm{sw}, \text { linear }}=$ $11.65-11.4=0.25 \mathrm{~V}$. This would mean that the full region approach

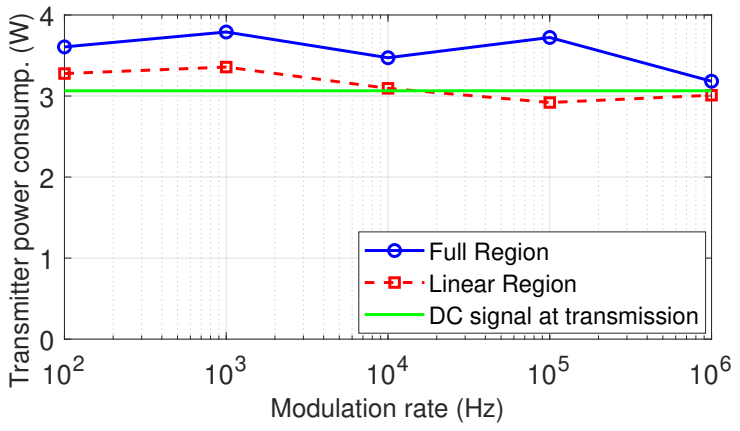

Figure 9: Transmitter power consumption versus modulation rate.

consumes 56 times larger than the linear region approach. However, as shown in Fig. 9, the total energy consumed by the full region approach is only 1.1 times larger than that in the linear region approach. Thus, we demonstrate here that most of the power is consumed for illumination purposes rather than for communication, and that is why VLC is a low-energy communication technology, as the LEDs are there for illuminating and only a little extra energy is needed to provide LEDs with communication capabilities.

To sum up, the extra energy that a full LED region approach involves in comparison to a linear LED region approach is negligible as long as we consider the important gains obtained in communication metrics demonstrated in Section 4.2.

\subsection{Energy harvesting through VLC}

The use of solar cells as a VLC receivers is attracting the interest of the research community, as both data reception and harvesting can perform simultaneously in order to create autonomous devices [8]. Here we explore how the transmission in the full LED region or linear LED region affects the harvesting efficiency. Fig. 10 shows the open-circuit voltage of the solar cell SLMD121H04L when employing both full region approach and linear region approach at transmission for different modulation rates. As explained before, temperature issues arise at low frequencies when transmitting at the full region which causes the signal average to decrease (Fig. 10a), and hence reduces the energy harvesting efficiency. Besides, if the temperature was controlled, the use of full LED region may saturate 


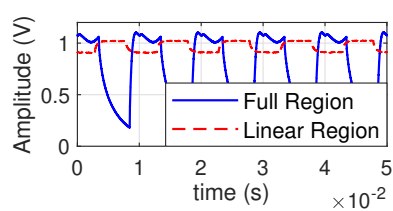

(a) $100 \mathrm{~Hz}$

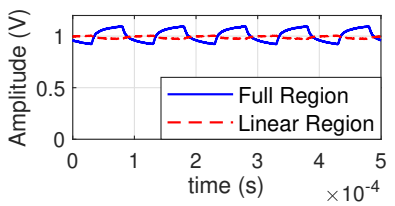

(c) $10 \mathrm{kHz}$

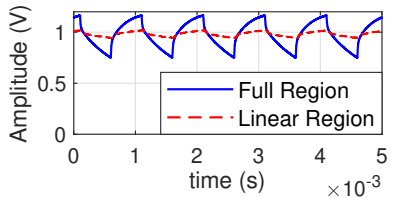

(b) $1 \mathrm{kHz}$

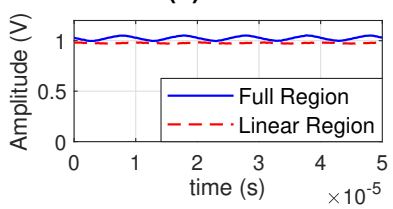

(d) $100 \mathrm{kHz}$

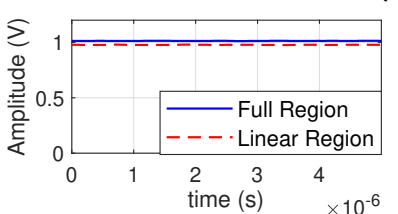

(e) $1 \mathrm{MHz}$
Figure 10: Received signal (voltage at the output of solar cell IXOLAR SLMD121H04L) with a distance of $1.5 \mathrm{~m}$ between transmitter and receiver for different modulation rates: (a) $100 \mathrm{~Hz}$; (b) $1 \mathrm{kHz}$; (c) $10 \mathrm{kHz}$; (d) $100 \mathrm{kHz}$; (e) $1 \mathrm{MHz}$.

the solar cell for high values. However, if temperature and saturation issues do not exist, both approaches provide similar signal average and then the energy harvested is the same as long as we work within the linear region of the solar cell and harvester chip efficiency curve. The slight difference in the average signal in Fig. 10d and $10 \mathrm{e}$ is due to an insignificant imbalance in the illuminance provided by both approaches. Finally, the use of full region approach allows demodulating the signal at high frequencies $(\sim 100 \mathrm{kHz})$, whereas the linear region approach loses communication capabilities due to its small peak-to-peak transmit illuminance swing and the slow speed response of solar cells.

\section{CONCLUSION}

In this paper, we have shown that working in the non-linear region of LEDs is possible and it brings benefits for VLC IoT applications that require to increase the communication range. We have compared the performance of LEDs modulated in the traditional linear region and our approach for operation in the full region. Both approaches providing the same illuminance were described, and the potential temperature issue when employing the full LED region at low modulation rates was discussed. Working in the full LED region was demonstrated to provide a much better communication performance than the approach that uses only the LED linear region: we obtain a gain of around $15 \mathrm{~dB}$ in the electrical received power and we achieve a larger range for the same SNR value. An extra power consumption is required at the LED transceiver in the infrastructure, but we have shown that the SNR at the receiver increases faster than the power consumption at the LED transmitter. In addition, most of the power is allocated for illumination purposes and through the electrical infrastructure. Finally, if a solar cell is used as receiver, the full region approach allows achieving larger rates.

\section{ACKNOWLEDGMENTS}

This work has been funded by the European Union's Horizon 2020 research and innovation programme under the Marie Skłodowska Curie grant agreement ENLIGHTEM No. 814215.

\section{REFERENCES}

[1] Cree XLAMP XHP35 LEDs. https://www.cree.com/led-components/media/ documents/ds-XHP35.pdf.

[2] Z. Ahmed, R. Singh, W. Ali, G. Faulkner, D. O'Brien, and S. Collins. A SiPM-based VLC receiver for Gigabit communication using OOK modulation. IEEE Photon. Technol. Lett., 32(6):317-320, 2020.

[3] J. Beysens, Q. Wang, A. Galisteo, D. Giustiniano, and S. Pollin. A cell-free networking system with visible light. IEEE/ACM Trans. Netw., 28(2):461-476, 2020.

[4] C. Chen, D. A. Basnayaka, and H. Haas. Downlink performance of optical attocell networks. F. Lightw. Technol., 34(1):137-156, 2016.

[5] X. Deng, Y. Wu, A. M. Khalid, X. Long, and J.-P. M. G. Linnartz. LED power consumption in joint illumination and communication system. Opt. Express, 25(16):18990-19003, Aug. 2017.

[6] H. Elgala, R. Mesleh, and H. Haas. An LED model for intensity-modulated optical communication systems. IEEE Photon. Technol. Lett., 22(11):835-837, 2010.

[7] A. Galisteo, D. Juara, and D. Giustiniano. Research in visible light communication systems with OpenVLC1.3. In Proc. IEEE WF-IoT, 2019.

[8] A. Galisteo, A. Varshney, and D. Giustiniano. Two to tango: Hybrid light and backscatter networks for next billion devices. In Proc. ACM MobiSys, 2020.

[9] Z. Ghassemlooy, W. Popoola, and S. Rajbhandari. Optical wireless communications: System and channel modelling with MATLAB, chapter 2. CRC Press, Inc., USA, 1st edition, 2012.

[10] C.-S. A. Gong, Y.-C. Lee, J.-L. Lai, C.-H. Yu, L. R. Huang, and C.-Y. Yang. The high-efficiency LED driver for visible light communication applications. Sci Rep, 6(1):30991-30991, 2016.

[11] T. Li, C. An, Z. Tian, A. T. Campbell, and X. Zhou. Human sensing using visible light communication. In Proc. ACM MobiCom, 2015.

[12] M. S. S. Mir, B. Genoves Guzman, D. Solanki, G. Marent, and D. Giustiniano. Poster: Integration between home automation and visible light communications. In Proc. ACM EWSN, 2020.

[13] N. O. Tippenhauer, D. Giustiniano, and S. Mangold. Toys communicating with LEDs: Enabling toy cars interaction. In Proc. IEEE CCNC, 2012.

[14] P. Wang, L. Feng, G. Chen, C. Xu, Y. Wu, K. Xu, G. Shen, K. Du, G. Huang, and $\mathrm{X}$. Liu. Renovating road signs for infrastructure-to-vehicle networking: A visible light backscatter communication and networking approach. In Proc. MobiCom, 2020.

[15] X. Xu, Y. Shen, J. Yang, C. Xu, G. Shen, G. Chen, and Y. Ni. PassiveVLC: Enabling practical visible light backscatter communication for battery-free IoT applications. In Proc. ACM MobiCom, 2017.

[16] M. Yamada and K. Stober. Adoption of light-emitting diodes in common lighting applications. Technical report, Chicago, USA, 2015.

[17] C. Zhang and X. Zhang. LiTell: robust indoor localization using unmodified light fixtures. In Proc. ACM MobiCom, 2016. 